\title{
Simulation Study on Evolutionary Game Model between Small Technology-Based Firms and Banks under Verification System
}

\section{Huafeng Chen Mu Zhang Lan Xiang}

School of Finance, Guizhou University of Finance and Economics, Guiyang Guizhou 550025, China

\begin{abstract}
In this paper, under the evolutionary game theory, the risk compensation funds are added to establish the evolutionary game model of the small technology-based firms and the bank loans under the verification system, and the evolutionary game results of the four different evolution strategies are studied by Matlab simulation. Finally, we give some suggestions of the choice of strategy and the formulation of loans.
\end{abstract}

\section{Keywords}

Evolutionary game model; small technology-based firms; risk compensation funds; verification system

\section{核查制度下科技型中小企业与银行间的 演化博弯模型及仿真研究}

陈华丰 张目 向岸 贵州财经大学金融学院, 贵阳 550025, 中国

摘要：本文在演化博弯理论下，加入风险补偿资金，建立了核查制度下科技型中小企业与银 行贷款演化博弯模型，并运用 Matlab 仿真研究了 4 种不同演化策略下模型演化博弯结果.最 后，对博弯双方的策略选择和贷款制定制定给出一些建议。

关键词: 演化博峦模型；科技型中小企业；风险补偿资金；核查制度；仿真

1. 引言

科技型中小企业作为提高综合国 力和国家竞争优势的重要源泉, 它的 发展关系整个国家经济的未来。与一 
般的中小企业相比，科技型中小企业 的最大区别是在于它主要从事高新技 术产品的研究开发、生产与销售, 拥 有较强的创新能力和较强的发展潜 力。我国政府一直积极探索如何通过 政策导向，发挥政府宏观调控的作 用，增强我国自主创新能力，使科技 创新成为㧼动我国经济增长的新动 力。因此，促进科技型中小企业快速 发展将增强我国的科技创新能力, 推 动我国经济的快速发展。但是科技型 中小企业具有高风险、规模小、有形 资产少、无形资产多和信用级别低等 特点, 导致了它融资十分困难。部分 学者在中小企业自身缺陷的方面做了 一些研究。例如,

银行作为主要放贷机构, 面对科 技型中小企业的一系列特点, 以及放 贷过程中的信息不对称和道德风险。 这使得银行产生 “惜贷”现象，使科 技型中小企业融资困难。余明桂, 潘 红波 (2008) ${ }^{[1]}$ 研究发现, 有政治关系的 企业比无政治关系的企业获得更多的 银行贷款和更长的贷款期限, 而且, 在 金融发展越落后、法治水平越低和政 府侵害产权越严重的地区, 政治关系的 这种贷款效应越显著。姬会英 $(2011)^{[2]}$ 研究发现由于金融机构贷款的发放程 序、经办环节等大致相同，而每户中 小企业平均获得的贷款规模大大低于 大企业，因而中小企业每笔贷款的经 营成本对金融机构来说相对较高。张 目, 周宗放 (2012) ${ }^{[3]}$ 利用 $\mathrm{k}-$ means 聚类算法从初始聚类中心开始, 得到 最终的聚类中心, 然后对信用等级进 行分区。从而实现了企业的信用评 级。刘金文 (2012) ${ }^{[4]}$ 发现中小企业外 部融资主要依靠银行贷款的情况下, 中 小企业信贷市场存在的“逆向选择”和" 道德风险”问题进一步加剧了中小企业 的资金短缺, 贷款难已经成为制约中小 企业发展的“瓶颈”。张晓玫, 钟祯 (2013) ${ }^{[5]}$ 基于上市中小企业 2008 年
银行贷款数据, 对我国银行规模和上市 中小企业贷款之间的关系进行了实 证。得出结论: 在中国, 小银行不应将 优质上市中小企业作为贷款对象。颜 白鹭（2015） ${ }^{[6]}$ 采用因子分析法, 建立 非均衡模型对中小企业融资供需情况 进行分析, 并运用 Fisher 判别研究了 中小企业银行贷款违约的影响因素。 研究发现, 无论是民间借贷还是银行贷 款, 都倾向于向规模更大的企业提供融 资; 民间借贷多、民间借贷利率高的 中小企业, 银行贷款违约的可能性更 大。

综上所述, 国内外学者对中小企 业融资问题的研究成功比较丰富, 但 对科技型中小企业融资问题研究的较 少。本讨论了在有限理性的前提下, 将演化博弯理论应用在科技型中小企 业与银行之间的博弯问题上, 建立了 核查制度下科技型中小企业与银行贷 款演化博亦模型, 对企业行为策略选 择与银行行为策略选择的互动机制进 行分析。旨在促进银行发放贷款, 科 技型中小企业能按时还款, 从而改善 科技型中小企业的融资难现状。

\section{2. 模型建立}

演化博亦理论的形成和发展大致 经历三个阶段: 首先, 生物学家从博 亦论中得到启示, 运用博亦论建构各 种生物竞争演化模型, 包括动物竞 争、性别分配以及植物的成长和发展 等: 接着, 生物学家根据生物演化的 自身规律, 对传统博亦论进行改造, 包括将传统博弯论中支付函数转化为 生物适应度函数、引入突变机制将传 统的纳什均衡精炼为演化稳定均衡以 及引入选择机制建构复制者动态模 型。随后, 鉴于演化博弃对传统博弃 的拓展, 经济学家又反过来借鉴生物 学家的思想, 将演化博亦运用到经济 
学中, 这又进一步推动演化博弯的发 展 $^{[7]}$ 。

\section{1. 核查制度下科技型中小企业与银行 贷款演化博弯模型}

演化博弯模型假设: (1)博弯中仅 有两个参与者: 科技型中小企业（和 银行, 博弯双方都是有限理性的。(2) 行为策略: 科技型中小企业的策略选 择集合为 $\mathrm{S}_{1}=\{$ 逾期违约, 按时还 款 $\}$; 银行的策略选择集合为 $\mathrm{S}_{2}=\{$ 核 查, 不核查\}。(3)行为策略采取的比 例: 科技型中小企业和银行博弯的初 始阶段，假设科技型中小企业采取逾 期违约的概率为 1-q, 采取按时还款的 概率为 $\mathrm{q}$; 银行采取贷款的概率为 $\mathrm{p}$, 采取不贷款的概率为 $1-p$ 。(4)参数假设 和基本解释： L: 科技型中小企业用于 项目投资需要从银行贷款的资金; $\mathrm{K}$ : 企业用于项目投资的自有资金； $\mathrm{a}$ ：科 技型中小企业投资项目成功时企业的 收益率, 失败时为 $0 ; \mathrm{r}$ ：银行的贷款 利率; $\mathrm{T}$ ：贷款期限； $\mathrm{i}$ ：银行的资金 成本率; $\mathrm{r}_{0}$ ：政府部门设立风险补偿资 金，在企业违约时给予放贷银行的 $\mathrm{r}_{0}$ 比例的补偿; $\mathrm{p}_{\mathrm{i}}$ ：科技型中小企业投资 项目成功的平均概率, 且 $0 \leq \mathrm{p}_{\mathrm{i}} \leq 1 ; \mathrm{C}$ : 银行监管过程中核查到科技型中小企 业在贷款后存在着骗贷的行为, 将对 科技型中小企业进行的惩罚; vL：银 行核查成本, 并假设 $\mathrm{vL}<\mathrm{C}$ 。假设银行 的贷后监管核查是 $100 \%$ 有效，只要银 行选择监管核查, 即表示银行可以准 确知晓科技型中小企业是否存在骗贷 违约的行为。

结合上述银行和科技型中小企业 在各种情况下的收益分析建立支付矩 阵，如表 1 所示。
表 1: 支付矩阵

科技型中小企业

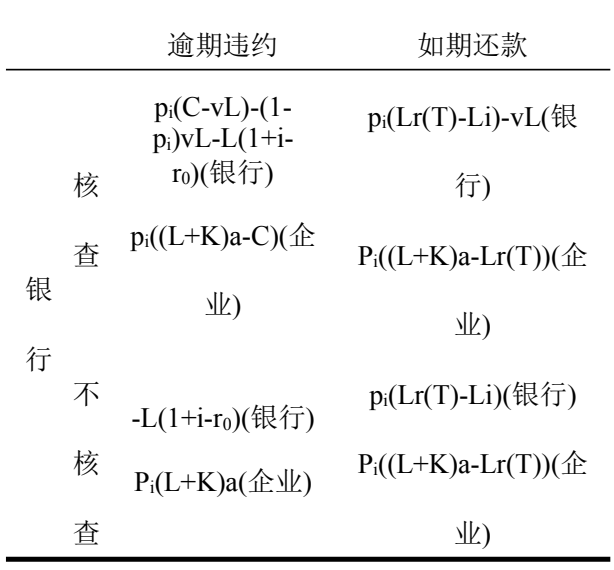

银行采取核查的期望收益、银行 采取不核查的期望收益、银行采取核 查和不核查策略的平均期望收益函数 $\mathrm{U}_{11} 、 \mathrm{U}_{12} 、 \mathrm{U}_{1}$ 分别为:

$\mathrm{U}_{11}=(1-\mathrm{q}) \quad\left(\mathrm{p}_{\mathrm{i}}(\mathrm{C}-\mathrm{vL})-\left(1-\mathrm{p}_{\mathrm{i}}\right) \mathrm{vL}-\mathrm{L}(1+\mathrm{i}-\right.$

$\left.\left.\mathrm{r}_{0}\right)\right)+\mathrm{q}\left(\mathrm{p}_{\mathrm{i}}(\mathrm{Lr}(\mathrm{T})-\mathrm{Li})-\mathrm{vL}\right)$;

$\mathrm{U}_{12}=(1-\mathrm{q}) \quad\left(-\mathrm{L}\left(1+\mathrm{i}-\mathrm{r}_{0}\right)\right)+\mathrm{q}\left(\mathrm{p}_{\mathrm{i}}(\operatorname{Lr}(\mathrm{T})-\right.$

Li) ) ;

$\mathrm{U}_{1}=\mathrm{p} \mathrm{U}_{11}+(1-\mathrm{p}) \mathrm{U}_{12}$

企业采取逾期违约的期望收益、

企业采取按时还款的期望收益、企业 采取逾期违约和按时还款策略的平均 期望收益函数 $U_{21} 、 U_{22} 、 U_{2}$ 分别为:

$$
\begin{aligned}
& \mathrm{U}_{21}=\mathrm{p}\left(\mathrm{p}_{\mathrm{i}}((\mathrm{L}+\mathrm{K}) \mathrm{a}-\mathrm{C})\right)+(1-\mathrm{p}) \\
& \quad(\mathrm{Pi}(\mathrm{L}+\mathrm{K}) \mathrm{a}) ; \\
& \mathrm{U}_{22}=\mathrm{p} \quad(\mathrm{Pi}((\mathrm{L}+\mathrm{K}) \mathrm{a}-\mathrm{Lr}(\mathrm{T})))+(1-\mathrm{p}) \\
& \quad(\mathrm{Pi}((\mathrm{L}+\mathrm{K}) \mathrm{a}-\mathrm{Lr}(\mathrm{T}))) ; \\
& \mathrm{U}_{2}=(1-\mathrm{q}) \mathrm{U}_{21}+\mathrm{qU}_{22}
\end{aligned}
$$

进而构建银行和企业策略的复制 者动态方程组:

$$
\begin{aligned}
& \frac{d p}{d t}=p\left(U_{11}-U_{1}\right)= \\
& p(1-p)\left((1-q)\left(p_{i} C-v L-q v l\right)\right) \\
& \frac{d q}{d t}=q\left(U_{22}-U_{2}\right)= \\
& q(1-q)\left(p p_{i} C-p_{i} \operatorname{Lr}(T)\right)
\end{aligned}
$$




\section{3. 模型仿真分析}

该复制者动态方程组的均衡点 $\mathrm{E}$ $(\mathrm{q}, \mathrm{p})$ 有 5 个局部均衡点, $\mathrm{E}_{1}(0$, $0), \mathrm{E}_{2}(1,0), \mathrm{E}_{3}(0,1), \mathrm{E}_{4}$ $(1,1), \mathrm{E}_{5}\left(\mathrm{q}^{*}, \mathrm{p}^{*}\right)$, 其中 $q^{*}=\frac{p_{i} C-v L}{p_{i} C}, p^{*}=\frac{\operatorname{Lr}(T)}{C}$, 且 $0<\frac{p_{i} C-v L}{p_{i} C}, \frac{\operatorname{Lr}(T)}{C}<1$

为了描述简便, 我们令 $\mathrm{a}=\mathrm{p}_{\mathrm{i}}(\mathrm{C}$ vL)-(1- $\left.p_{i}\right) v L-L\left(1+i-r_{0}\right), \quad b=p_{i}(\operatorname{Lr}(T)-L i)-$ $\mathrm{vL}, \quad \mathrm{c}=-\mathrm{L}\left(1+\mathrm{i}-\mathrm{r}_{0}\right), \quad \mathrm{d}=\mathrm{pi}(\mathrm{Lr}(\mathrm{T})-\mathrm{Li})$, $\mathrm{e}=\mathrm{p}_{\mathrm{i}}(\mathrm{La}-\mathrm{C}), \quad \mathrm{f}=\mathrm{p}_{\mathrm{i}} \mathrm{L}(\mathrm{a}-\mathrm{r}(\mathrm{T})), \quad \mathrm{g}=\mathrm{p}_{\mathrm{i}} \mathrm{La}$, $\mathrm{h}=\mathrm{p}_{\mathrm{i}} \mathrm{L}(\mathrm{a}-\mathrm{r}(\mathrm{T}))$ 。根据 $\mathrm{a} 、 \mathrm{~b} 、 \mathrm{c} 、 \mathrm{~d} 、 \mathrm{e} 、$ $f 、 g 、 h$ 的相对大小，对复制系统在平 衡点附近进行线性稳定性分析, 可以 得到演化博弯模型的各种均衡情况 ${ }^{[8]}$ 。 因模型假设易知, $b<d, g>h$ 。结合模 型假设后，分析发现复制系统有 4 种 演化情况。

\section{1. 演化情况 1}
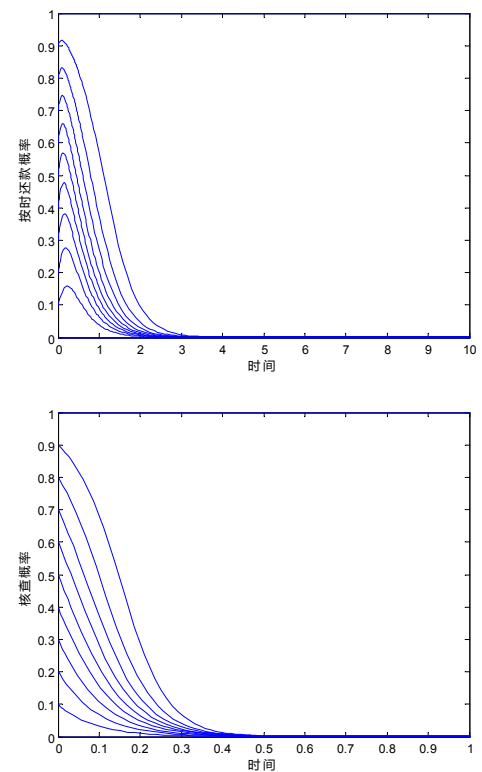

图 1: 演化情况 $1 （ \mathrm{p}_{\mathrm{i}}=50 \%, \mathrm{C}=30, \mathrm{vL}=20$, $\mathrm{L}=100, \mathrm{r}(\mathrm{T})=5 \%$ )
当模型参数满足 $\mathrm{a}<\mathrm{c}, \mathrm{b}<\mathrm{d}, \mathrm{e}<\mathrm{f}$, $\mathrm{g}>\mathrm{h}$ 时, $\mathrm{E}_{1}(0,0)$ 是稳定结点, $\mathrm{E}_{2}$ $(1,0)$ 和 $\mathrm{E} 4(1,1)$ 是鞍点, $\mathrm{E}_{3}$ $(0,1)$ 是不稳定结点。即在这种情 况下，科技型中小企业会采取“逾期违 约”策略, 银行会采取“不核查”策略。 设置参数如图 1, 进行仿真模拟, 得图 1。所得仿真结果与稳定性分析中的结 果一致。如图分析, 银行策略的收敛 速度较快, 在 0 。5 个单位时间内所有 博弯中“核查”策略已经趋向于 0 。企业 策略的收玫速度较慢, 在 3.5 个单位时 间内所有博弯中“按时还款”策略趋向 于 0 。且 $\mathrm{q}$ 和 $\mathrm{p}$ 的起始博弯值设置的越 小, 它趋向于稳定的速度就越快。还 可以发现，企业在博弯初始阶段“按时 还款”的概率有一段上升的过程, 随后 下降趋近于 0 。可能是因为银行策略收 玫的速度较快, 在 0.5 个单位时间内收 敛至稳定策略“不核查”，以至于企业 选择“逾期违约”以便获得更大的收 益。

\section{2. 演化博弯 2}
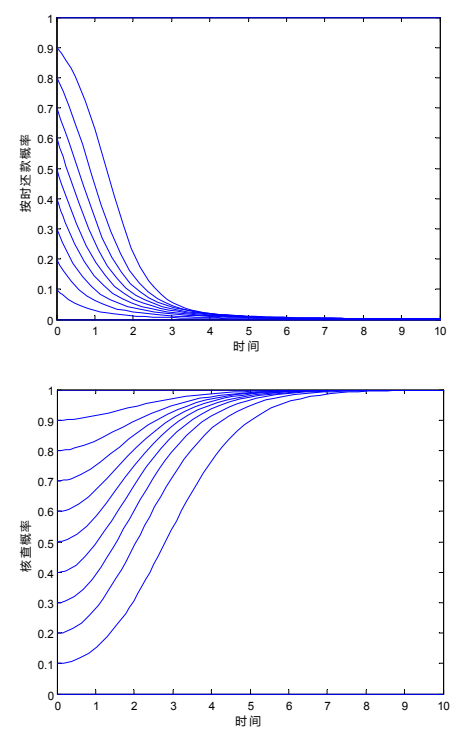

图 2: 演化情况 2（ $\mathrm{p}_{\mathrm{i}}=50 \%, \mathrm{C}=4, \mathrm{vL}=1$, $\mathrm{L}=100$, $\mathrm{r}(\mathrm{T})=5 \%$ ) 
当模型参数满足 $a>c, b<d, e>f, g>h$ 时, $\mathrm{E}_{3}(0,1)$ 是稳定结点, $\mathrm{E}_{1}(0$, $0)$ 和 $E_{2}(1,0)$ 鞍点, $E_{4}(1,1)$ 是 不稳定结点, 即在这种情况下, 科技 型中小企业会采取“逾期违约”策略, 银行会采取“核查”策略。设置参数如 图 2, 进行仿真模拟, 得图 2。所得仿 真结果与稳定性分析中的结果一致。 如图分析, 企业与银行博弯策略收玫 的时间相近，因此没有出现上一种在 博弯初始阶段稳定策略概率向相反收 敛方向运动的过程。

\section{3. 演化情况 3}
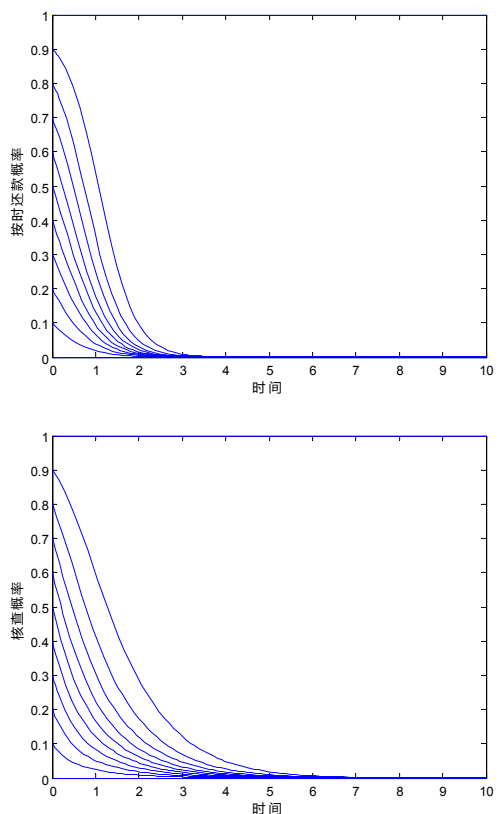

图 3: 演化情况 3（ $\mathrm{p}_{\mathrm{i}}=50 \%, \mathrm{C}=4, \mathrm{vL}=3$, $\mathrm{L}=100, r(\mathrm{~T})=5 \%)$

当模型参数满足 $a<c, b<d, e>f$, $\mathrm{g}>\mathrm{h}$ 时, $\mathrm{E}_{1}(0,0)$ 是稳定结点, $\mathrm{E}_{2}$ $(1,0)$ 和 $\mathrm{E}_{3}(0,1)$ 鞍点, $\mathrm{E}_{4}(1$, 1) 是不稳定结点。即在这种情况下, 科技型中小企业会采取“逾期违约”策 略, 银行会采取“不核查”策略。设置 参数如图 3, 进行仿真模拟, 得图 3。 所得仿真结果与理论结果一致。如图
分析, 企业策略的收玫速度较快, 在 3。 5 个单位时间内所有博弯中“按时还 款”策略的概率已经趋向于 0 。且 $X$ 得 起始博弯值设置的越小, 它趋向于稳 定的速度就越快。银行的收敛速度相 对较慢, 大约在 7 个单位时间内所有 博弯中“核查”策略已经趋向于 0 。且起 始博弯值设置的越小, 它趋向于稳定 的速度就越快。在此种参数设置条件 下，科技型中小企业和银行收敛至稳 定的时间相差不大，但仍有先后。

\section{4. 演化情况 4}

当模型参数满足 $a>c, b<d, e<f$, $\mathrm{g}>\mathrm{h}$ 时, 在这种情况下, $\mathrm{E}_{1}(0,0) \mathrm{E}_{2}$ $(1,0), \mathrm{E}_{3}(0,1), \mathrm{E}_{4}(1,1)$ 是鞍点, $E_{5}\left(q^{*}, p^{*}\right)$ 是中心, 其中 $q^{*}=\frac{p_{i} C-v L}{p_{i} C}, p^{*}=\frac{\operatorname{Lr}(T)}{C}$ 。科技型 中小企业和银行博弯的稳定策略概率 在中点 $\mathrm{E}_{5}\left(\mathrm{q}^{*}, \mathrm{p}^{*}\right)$ 附近震荡, 震荡 的中心就是 $\mathrm{E}_{5}\left(\mathrm{q}^{*}, \mathrm{p}^{*}\right)$ 。设置参数 如图 4, 进行仿真模拟, 得图 4。如图 分析, $\mathrm{q}$ 与 $\mathrm{p}$ 共同的博弯演化过程呈环 形, 没有稳定点。 $\mathrm{p}$ 取值的变化会影响 $\mathrm{q}$ 取值的变化, $\mathrm{q}$ 取值的变化又会影响 $\mathrm{p}$ 取值的变化。为了自身利益的最大 化，科技型中小企业的策略选择会根 据银行的策略选择而不断调整, 银行 的策略选择也会根据科技型中小企业 的策略选择而不断调整。 $\mathrm{q}$ 的取值先增 大再逐渐减小, 之后一直如此循环。 $p$ 的取值先减小至 0 , 然后保持一段时间 的稳定再逐渐增大, 之后一直如此循 环。从图中还能发现, 起始博恋值越 小, 博弯过程中策略的最大概率值也 越小, 且循环周期也越短。 


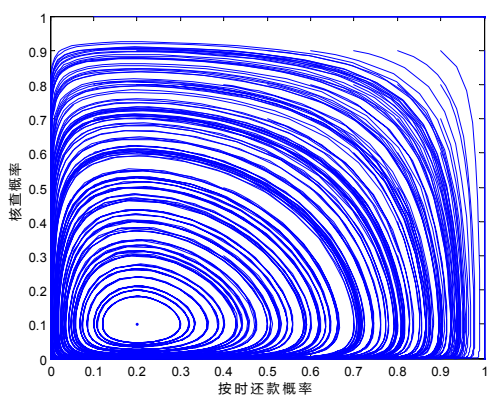

图 4: 演化情况 4（ $\mathrm{p}_{\mathrm{i}}=50 \%, \mathrm{C}=50, \mathrm{vL}=20$, $\mathrm{L}=100, \mathrm{r}(\mathrm{T})=5 \%)$

\section{4. 结论}

本文将演化博弯理论应用在银行 与科技型中小企业之间的博弯问题 上，对科技型中小企业贷款过程中， 企业行为策略选择与银行行为策略选 择的互动机制进行分析。结果发现, 在演化情况 $1 、 2 、 3$ 中，科技型中小 企业的策略选择都是“逾期违约”。在 演化情况 4 中, 科技型中小企业和银 行的策略选择围绕中心 $\mathrm{E}_{5}\left(\mathrm{q}^{*}, \mathrm{p}^{*}\right)$ 震荡, 并逐渐趋近于中心。并发现在 这种演化情况下，科技型中小企业投 资项目成功的平均概率 $\left(\mathrm{p}_{\mathrm{i}}\right)$ 、对科技 型中小企业进行的惩罚（C）与科技型 中小企业“逾期违约”的概率呈反比。 银行核查成本 (vL) 与科技型中小企 业“逾期违约”的概率呈正比。银行贷 款利息 $\mathrm{r}$ （T）与银行“核查”概率呈正 比。

根据上述结论, 本文建议(1)提升 科技型中小企业投资项目成功的平均 概率, 政府应该对科技型中小企业投 资项目提供一定的帮助。(2)加大违约 时对科技型中小企业的惩罚力度，以 使科技型中小违约时所要付出的代价 更大，以降低科技型中小企业选择“预 期违约”的概率。

\section{致谢}

基金项目：本文获得 2017 年度贵 州财经大学校级科研项目（青年项 目）“贵州大数据产业集聚效应及金融 支持体系研究”; 贵州财经大学 2016 年度在校学生资助项目 “贷款风险补 偿机制下科技型中小企业信用风险感 知及个主体间博弯仿真研究” 资助。

\section{参考文献}

[1] 余明桂, 潘红波. 政治关系、制度 环境与民营企业银行贷款 $[\mathrm{J}]$. 管理 世界，2008(8)：9-21。

[2] 姬会英.当前我国中小企业融资难 的原因及对策探析 $[\mathrm{J}]$. 特区经 济,2011,(2):219-221.

[3] Zhang M, Zhou Z. A Credit Rating Model for Enterprises Based on Projection Pursuit and K-Means Clustering Algorithm[J]. Journal of Risk Analysis \& Crisis Response, 2012, 2(2): 131.

[4] 刘金文. 基于中小企业、银行和政 府三方动态博峦的中小企业贷款难 题研究 $[\mathrm{J}]$. 金融监管研究, 2012(9):69-84.

[5] 张晓玫, 钟祯. 银行规模与上市中小 企业贷款一一基于中国上市中小企 业银行贷款数据的经验研究 $[\mathrm{J}]$. 南 开经济研究, 2013(2):94-111.

[6] 颜白鹭. 银行贷款、民间借贷与中 小企业融资——基于对非上市中小 企业调查数据的研究 $[\mathrm{J}]$. 金融监管 研究, 2015(5):63-74.

[7] 黄凯南.演化博弯与演化经济学 [J]. 经济研究,2009(2):132-145.

[8] 孙庆文,陆柳,严广乐,等.不完全信息 条件下演化博弯均衡的稳定性分析 [J]. 系统工程理论与实践, 2003, 23(7):11-16. 